\title{
POLLUTION, POLITICS, and POWER
}



Thomas O. McGarity

\section{POLLUTION, POLITICS, and POWER}

The Struggle for Sustainable Electricity 
Copyright $\odot 2019$ by the President and Fellows of Harvard College All rights reserved

Printed in the United States of America

First printing

Jacket design: Lisa Roberts

Jacket photograph: amygdala_imagery | Getty Images

9780674242807 (EPUB)

9780674242814 (MOBI)

$9780674242791(\mathrm{PDF})$

The Library of Congress has cataloged the printed edition as follows:

Names: McGarity, Thomas O., author.

Title: Pollution, politics, and power : the struggle for sustainable electricity / Thomas O. McGarity.

Description: Cambridge, Massachusetts : Harvard University Press, 2019. | Includes bibliographical references and index.

Identifiers: LCCN 2019006756 | ISBN 9780674545434 (hardcover : alk. paper)

Subjects: LCSH: Electric utilities-United States-History. | Electric power-United States-History. | Environmental policy-United States-History. | Renewable energy sources-United StatesHistory. | Coal trade-United States-History. | Coal-fired power plants-United States-History.

Classification: LCC HD9685.U5 M3333 2019 | DDC 333.793/20973dc23 LC record available at https://lccn.loc.gov/2019006756 
This book is dedicated to my grandsons, Brendan, William, and Ryan 
